\title{
Stereoselective Synthesis of Southern Fragment of Hantupeptin A
}

\author{
Avula Srinivas,* Malladi Sunitha and Chakunta Govind Rao \\ Department of Chemistry, Vaagdevi Degree \& PG CollegeKishanpura, Warangal, Telangana, India 506001 \\ *Corresponding author: E-mail: avula.sathwikreddy@gmail.com
}

Received: 04-02-2016

\begin{abstract}
The stereoselective synthesis of the southern fragment (C21-C41) of Hantupeptin A is described. The required stereochemistry of $\beta$-hydroxy- $\alpha$-methyl acid unit was accomplished through the Aldol reaction using Evan's chiral auxiliary followed by the installation of the terminal alkyne with Ohira-Bestmann reagent.
\end{abstract}

Keywords: Hantupeptin A, Aldol Reaction, Stereoselective synthesis, Peptide; Evan's auxiliary

\section{Introduction}

Novel bioactive metabolites are emerging as an important source of pharmacologically active compounds or promising lead structures in drug discovery. ${ }^{1-5}$ Naturally occurring cyclic peptides ${ }^{6}$ have come within this class possessing diverse biological activities like immunosuppressant, antibiotic, antifungal, anti-inflammatory and anticancer effects. ${ }^{7}$ Indeed, marine organisms such as algae, sponges, and coelenterates became an exceptional source of these natural products. Since the discovery of the didemnins, this class of natural products continues to stimulate active research in synthetic and medicinal chemistry, as well as in clinical oncology and cell biology. ${ }^{8}$ In 2009 , Tan and co-workers have isolated a new cyclodepsipeptide, hantupeptin A (1) from the marine cyanobacterium Lyngbya majuscula. The hantupeptin A (1) has exhibited cytotoxicity against MOLT-4 leukemia cells and MCF-7 breast cancer cells with $\mathrm{IC}_{50}$ values of 32 and $4.0 \mu \mathrm{M}$, respectively. ${ }^{9}$ The extensive spectral studies and advanced chiral techniques have revealed the planar structure as well as the absolute configuration of $\mathbf{1}$. Structurally, compound $\mathbf{1}$ is a 19-membered cyclic tetrapeptide, which consists of ?ve $\alpha$-amino/hydroxy acid residues, including phenyl lactic acid, proline, $N$-methylvaline, valine, $N$-methylisoleucine and a $\alpha$-methyl- $\beta$-hydroxy acid unit with an alkyne at the terminal end of the molecule. The stereochemistry at the hydroxyl group attached carbon (C-35) of an unusual hydroxy acid, 3-hydroxy-2methyloctynoic acid (Hmoya) unit $\mathbf{4}$ was determined as $S$ using the Mosher's analysis. However, the stereochemi- stry at the methyl group attached carbon (C-34) was not reported at that point of time.

Later, in 2010, the same group has isolated hantupeptins B (2) and C (3) along with $\mathbf{1}$ from the organic extracts of the same marine cyanobacterium. ${ }^{10}$ The only structural difference among these molecules is the degree of unsaturation in the unusual amino acid part, where $\mathbf{1}$ is having a terminal alkyne functionality, $\mathbf{2}$ has an alkene and $\mathbf{3}$ is without any unsaturation in its structure (Figure 1). Compounds $\mathbf{2}$ and $\mathbf{3}$ also showed moderate in vitro cytotoxicity against MOLT-4 (leukemic) and MCF-7 (breast cancer) cell lines. In the study of the re-isolation of compound $\mathbf{1}$, the relative stereo chemistry at C-34 carbon of Hmoya unit was determined as $R$ by the rigorous NMR experiments. Very recently, the absolute configuration of

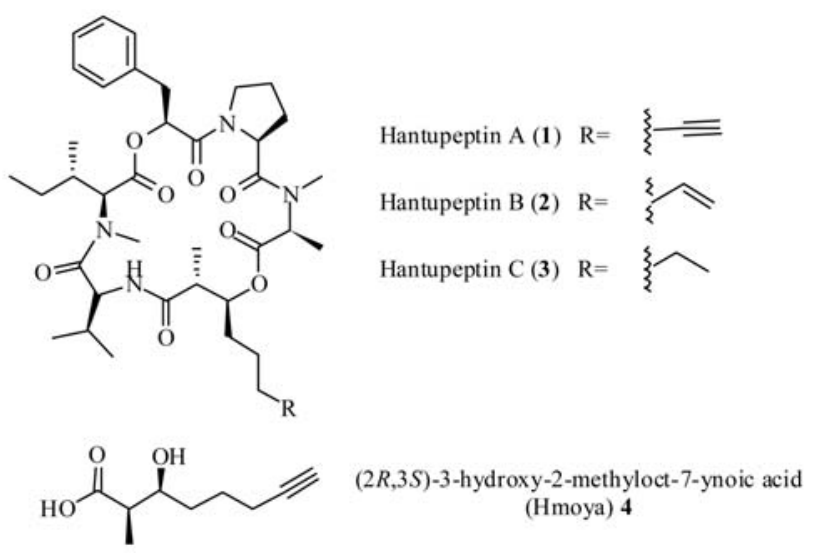

Figure 1: Structures of Hantupeptins A-C. 
Hmoya unit of hantupeptin C (3) was assigned as $(2 R, 3 S)$ based on the retention times of the Mosher ester derivative standards by RPLC-MS. ${ }^{11}$ Till date, no synthetic efforts have been reported in the literature for these molecules. In continuation of our interest on the synthesis of biologically active molecules, ${ }^{12}$ we have reported the synthesis of hantupeptin A C21-C41 fragment with the unusual component, Hmoya residue as a part of it. The Hmoya unit $\mathbf{4}$ is also present in a number of marine-derived compounds, such as onchidin B, kulomo'opunalide-1, kulomo'opunalide-2, and trungapeptin A.

\section{Results and Discussion}

From the retrosynthetic outlook (Scheme 1), the desired molecule was envisioned to be obtained from the key intermediate 5, which, in turn may be built from $\mathbf{6}$ through oxidation followed by Ohira-Bestmann reaction. Compound 6 could be obtained by coupling compound 7 with an amine, derived from compound $\mathbf{8}$. The stereocentres in compound $\mathbf{7}$ could be achieved through Evan's syn aldol protocol followed by a reductive etherification. The stereochemistry in compound $\mathbf{8}$ was achieved from the natural amino acids $L$-isoleucine and $L$-valine.

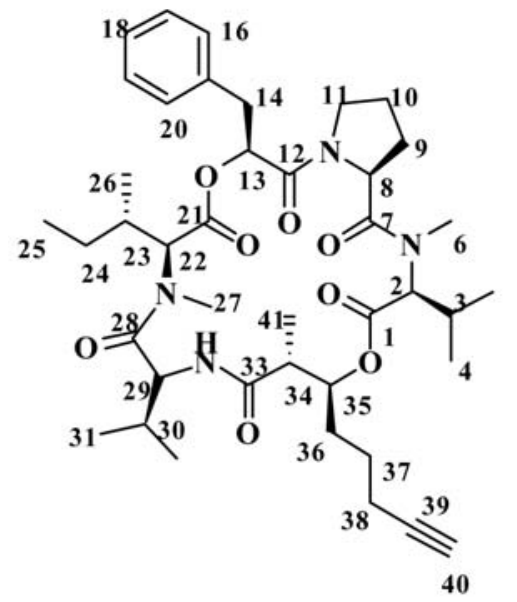

Hantupeptin A, 1

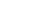

Preparation of the acid 7. The synthesis began with Evan's aldol reaction between the aldehyde $\mathbf{1 0}$ and oxazolidinone 9. Aldehyde $\mathbf{1 0}$ was prepared in two steps from the inexpensive 1,5-pentanediol following the reported procedure. ${ }^{13}$ The other, desired $(R)$-4-benzyl-3-propionyloxazolidin-2-one 9 was also smoothly obtained using well documented literature protocol. ${ }^{14}$ The di- $n$-butylboron triflate mediated Aldol reaction between compounds 9 and $\mathbf{1 0}$ furnished the syn-product $\mathbf{1 1}$ in $83 \%$ yield (Scheme 2). Protection of the secondary hydroxy group of the compound $\mathbf{1 1}$ as TBS-ether $\mathbf{1 2}$ was achieved in $87 \%$ yield by exposing to TBSCl/Imidazole in $\mathrm{CH}_{2} \mathrm{Cl}_{2}$ at room temperature for $18 \mathrm{~h}$. Compound 12 was then treated with sodium borohydride in $\mathrm{THF} / \mathrm{pH} 7$ buffer at room temperature for the reductive removal of the auxiliary to provide alcohol $\mathbf{1 3}$. The primary hydroxyl group of compound 13 was oxidized to a carboxylic acid using (bisacetoxyiodo)benzene (BAIB) / 2,2,6,6-tetramethyl-1piperidinyloxy free radical (TEMPO) in $\mathrm{CH}_{2} \mathrm{Cl}_{2} / \mathrm{pH} 7$ buffer to obtain the acid fragment 7 in $89 \%$ yield.

Preparation of compound 8 . The synthesis of compound $\mathbf{8}$ was commenced by carrying out the preparation of the known $N$-(tert-butoxycarbonyl)- $N$-methyl- $L$-isoleucine 14 from $L$-isoleucine using the literature procedure. ${ }^{15}$ Esterification of the acid $\mathbf{1 4}$ with allyl bromide was clean-

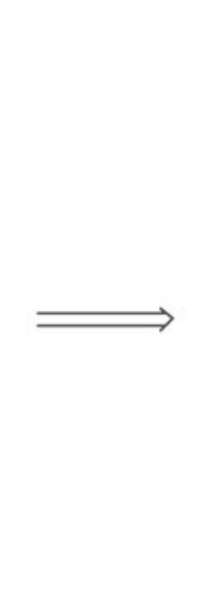<smiles>C#CCCC[C@H](O[Sb](=O)(=O)O[C@H](C)[C@@H](C)C(=O)N[C@H](C(=O)N(C)[C@H](C(=O)OCC=C)[C@@H](C)CC)C(C)C)C(C)C</smiles><smiles>C=CCOC(=O)[C@@H]([C@@H](C)CC)N(C)C(=O)[C@H](NC(=O)[C@H](C)[C@H](CCCCO)O[Na])C(C)C</smiles><smiles>C=CCCCCCO</smiles><smiles>C[C@H](C(=O)O)[C@H]([OH2+])CCCCOC(=O)[O-]</smiles><smiles>C=CCOC(=O)[C@@H]([C@@H](C)CC)N(C)C(=O)[C@@H](NC(=O)c1ccccc1)C(C)C</smiles> 


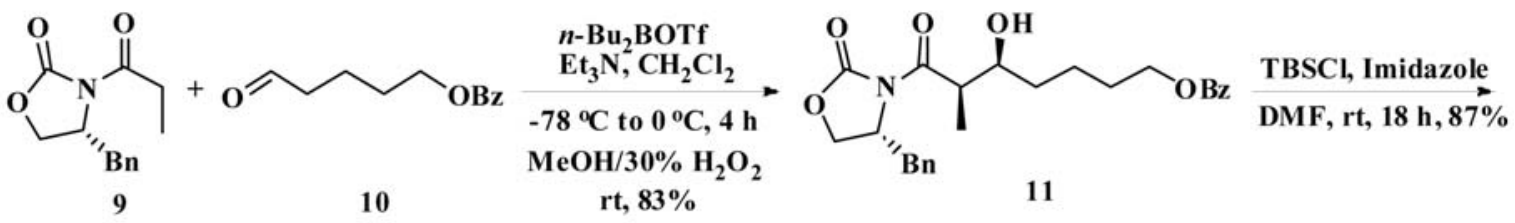<smiles>C[C@H](O[13CH3])[C@@H](C)C(=O)N1C(=O)OC[C@H]1Br</smiles>

12

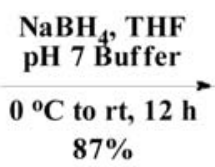

$87 \%$

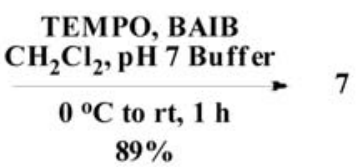

Scheme 2: Synthesis of the acid fragment 7.

ly achieved by using $\mathrm{K}_{2} \mathrm{CO}_{3}$ in DMSO solvent at room temperature, to get the ester $\mathbf{1 5}$ in $80 \%$ yield. The required amide $\mathbf{8}$ was prepared from $\mathbf{1 5}$ by two transformations. First treating with TFA in $\mathrm{CH}_{2} \mathrm{Cl}_{2}$ to get secondary amine in good yields and used as such for the next step without further purification. Later, the crude amine, prepared from 15 was coupled with (tert-butoxycarbonyl)- $L$-valine under standard reaction conditions in the presence of 1-hydroxy7-azabenzotriazole (HOAt) and 1-[bis(dimethylamino) methylene]-1H-1,2,3-triazolo[4,5- $b$ ]pyridinium-3-oxide hexafluorophosphate (HATU) as coupling agents and $\mathrm{N}, \mathrm{N}$-diisopropyl ethylamine (DIPEA) as the base in anhydrous $\mathrm{CH}_{2} \mathrm{Cl}_{2}$ as the solvent at room temperature for $8 \mathrm{~h}$ to acquire the desired dipeptide fragment 8 in $84 \%$ yield (Scheme 3).
Construction of C21-C41 segment from 7 and 8. With the successful completion of the desired fragments $\mathbf{7}$ and $\mathbf{8}$, the attention was turned to couple them to give the di-amide 16. For that, intially the boc protection of compound 8 was removed by using TFA in $\mathrm{CH}_{2} \mathrm{Cl}_{2}$ at 0 ${ }^{\circ} \mathrm{C}$ and then coupled with the compound $\mathbf{7}$ under HATU/HOAt conditions at room temperature, to obtain the required product $\mathbf{1 6}$ in $85 \%$ yield. The resulting compound $\mathbf{1 6}$ was hydrolyzed with potassium carbonate in methanol to give the primary alcohol 6 in $75 \%$ yield which upon the oxidation with TPAP/NMO to the aldehyde followed by the treatment with Ohira-Bestmann reagent gave the targeted terminal alkyne product 5 in $92 \%$ yield (Scheme 4 ).

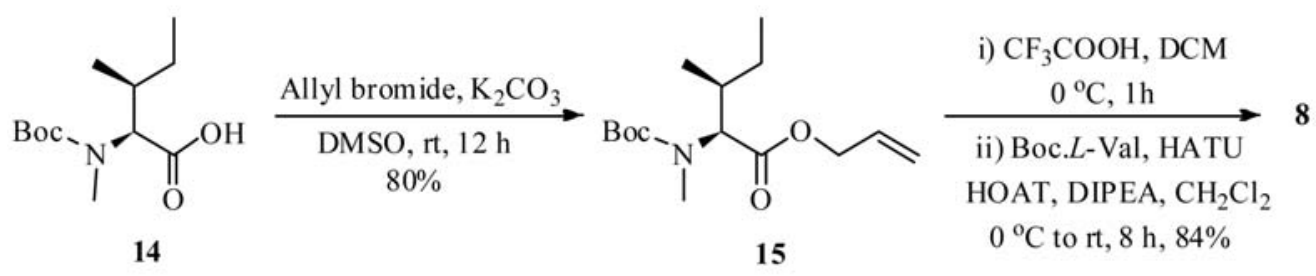

Scheme 3: Synthesis of the fragment 8.
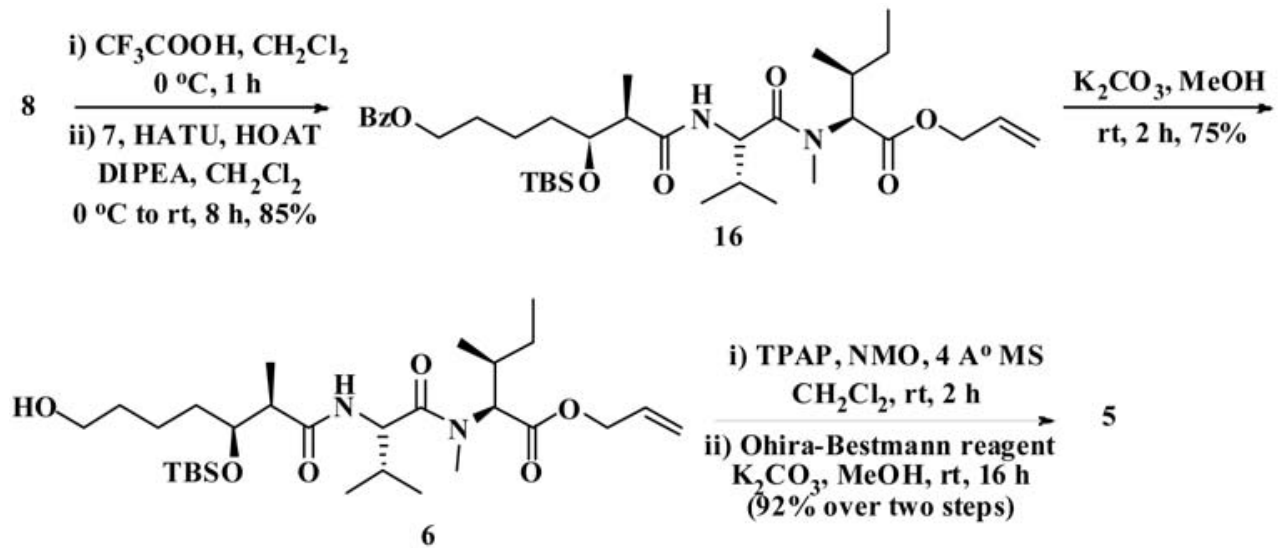

i) TPAP, NMO, $4 \mathrm{~A}^{\circ} \mathrm{MS}$

$\mathrm{CH}_{2} \mathrm{Cl}_{2}, \mathrm{rt}, 2 \mathrm{~h}$

ii) Ohira-Bestmann reagent

$\mathrm{K}_{2} \mathrm{CO}_{3}, \mathrm{MeOH}, \mathrm{rt}, 16 \mathrm{~h}$

(92\% over two steps)

Scheme 4: Construction of the C21-C41 building block. 


\section{Experimental}

NMR spectra were recorded in $\mathrm{CDCl}_{3}$ on Bruker AM-300 (300 MHz) spectrometer at ambient temperature. Chemical shifts are reported in ppm relative to TMS as internal standard and coupling constants are reported in $\mathrm{Hz}$. FTIR spectra were recorded on a Nicolet FT-IR 400 spectrometer in $\mathrm{KBr}$ or as neat. Optical rotations were measured on an Perkin-Elmer 141 polarimeter by using a $2 \mathrm{~mL}$ cell with a path length of $1 \mathrm{dm}$ with $\mathrm{CHCl}_{3}$ or $\mathrm{CDCl}_{3}$ as solvent. Low resolution mass spectra were obtained on VG 70-70H or LC/MSD trap SL spectrometer operating at $70 \mathrm{eV}$ using direct inlet system. High resolution mass spectra (HRMS) were recorded on an Agilent Technologies 6510 Q-TOF spectrometer. Technical-grade EtOAc and hexanes used for column chromatography were distilled before use. All the reagents and solvents were of reagent grade and used without further purification unless otherwise stated.

(5S,6R)-7-((R)-4-Benzyl-2-oxooxazolidin-3-yl)-5-hydro xy-6-methyl-7-oxoheptyl benzoate (11): To a stirred solution of acyl oxazolidinone $\mathbf{9}^{14}(1.04 \mathrm{~g}, 4.45 \mathrm{mmol})$ in $\mathrm{CH}_{2} \mathrm{Cl}_{2}(10 \mathrm{~mL})$ was added dropwise $n-\mathrm{Bu}_{2} \mathrm{BOTf}(1.0 \mathrm{M}$ in $\mathrm{CH}_{2} \mathrm{Cl}_{2}, 4.67 \mathrm{~mL}, 4.67 \mathrm{mmol}$ ) and stirred for $10 \mathrm{~min}$. $i$ - $\operatorname{Pr}_{2} \mathrm{NEt}(0.93 \mathrm{~mL}, 5.34 \mathrm{mmol})$ was then added dropwise and the reaction was stirred at the same temperature for $1 \mathrm{~h}$. The mixture was cooled to $-78^{\circ} \mathrm{C}$ before a solution of aldehyde $\mathbf{1 0}^{12}$ (1.02 g, $4.95 \mathrm{mmol}$ ) in $\mathrm{CH}_{2} \mathrm{Cl}_{2}(15 \mathrm{~mL})$ was added dropwise via cannula. Stirring was continued at -78 ${ }^{\circ} \mathrm{C}$ for $3 \mathrm{~h}$ before gradually warming to $0{ }^{\circ} \mathrm{C}$. The reaction mixture was stirred for additional $3 \mathrm{~h}$ at $0{ }^{\circ} \mathrm{C}$ and then quenched by the addition of $0.1 \mathrm{M} \mathrm{pH} 7$ phosphate buffer $(7.5 \mathrm{~mL})$ followed by $\mathrm{MeOH}(10 \mathrm{~mL})$ at $0{ }^{\circ} \mathrm{C}$. After stirring for $5 \mathrm{~min}$, a solution of $30 \%$ aqueous $\mathrm{H}_{2} \mathrm{O}_{2}(7.5 \mathrm{~mL})$ in Me$\mathrm{OH}(15 \mathrm{~mL})$ was added dropwise and stirred at the same temperature for $1 \mathrm{~h}$ before being concentrated under reduced pressure. The residue was diluted with $\mathrm{Et}_{2} \mathrm{O}$, the phases were separated and the aqueous phase extracted with $\mathrm{Et}_{2} \mathrm{O}$. The combined organic phase was washed with brine, dried over $\mathrm{Na}_{2} \mathrm{SO}_{4}$, filtered and concentrated under reduced pressure. Flash chromatography over silica gel (35\% ethyl acetate in pet. ether) gave $\mathbf{1 1}(1.63 \mathrm{~g}, 83 \%)$ as a viscous, colorless oil. IR $\left(\mathrm{CHCl}_{3}\right): v$ 3250, 2936, 1780, 1710, 1453, 1386, 1277, 1214, 1146, 763, $506 \mathrm{~cm}^{-1}$; ${ }^{1} \mathrm{H}$ NMR $(300$ $\left.\mathrm{MHz}, \mathrm{CDCl}_{3}\right): \delta 8.10-7.98(\mathrm{~m}, 2 \mathrm{H}), 7.55(\mathrm{t}, J=7.42 \mathrm{~Hz}$, $1 \mathrm{H}), 7.43(\mathrm{t}, J=7.5 \mathrm{~Hz}), 7.39-7.24(\mathrm{~m}, 3 \mathrm{H}), 7.20(\mathrm{~d}, J=5.6$ $\mathrm{Hz}, 2 \mathrm{H}), 4.71$ (ddt, $J=10.4,6.9,3.3 \mathrm{~Hz}, 1 \mathrm{H}), 4.33(\mathrm{t}, J=6.5$ $\mathrm{Hz}, 2 \mathrm{H}), 4.28-4.13(\mathrm{~m}, 2 \mathrm{H}), 4.04-3.93(\mathrm{~m}, 1 \mathrm{H}), 3.77$ (qd, $J$ $=7.0,2.7 \mathrm{~Hz}, 1 \mathrm{H}), 3.25(\mathrm{dd}, J=13.4,3.2 \mathrm{~Hz}, 1 \mathrm{H}), 2.95(\mathrm{~s}$, $1 \mathrm{H}), 2.79(\mathrm{dd}, J=13.4,9.4 \mathrm{~Hz}, 1 \mathrm{H}), 1.87-1.42(\mathrm{~m}, 6 \mathrm{H})$, $1.27(\mathrm{~d}, J=7.1 \mathrm{~Hz}, 3 \mathrm{H}) ;{ }^{13} \mathrm{C}$ NMR $\left(75 \mathrm{MHz}, \mathrm{CDCl}_{3}\right): \delta$ $178.0,166.8,153.3,135.2,133.1,130.0,129.0,128.0$, 127.3, 71.6, 66.2, 65.1, 55.1, 42.2, 38.2, 33.0, 28.5, 23.0, 10.4; HRMS (ESI): $\mathrm{m} / \mathrm{z}$ calculated for $\mathrm{C}_{25} \mathrm{H}_{30} \mathrm{O}_{6} \mathrm{~N}:[\mathrm{M}+\mathrm{H}]^{+}$ 440.2067, found 440.2044; $[\alpha]_{\mathrm{D}}{ }^{25}=+29.0\left(\right.$ c 1.62, $\left.\mathrm{CDCl}_{3}\right)$.
(5S,6R)-7-((R)-4-Benzyl-2-oxooxazolidin-3-yl)-5-(tertbutyldimethylsilyloxy)-6-methyl-7-oxoheptyl benzoate (12): To a stirred solution of $\mathbf{1 1}(1.60 \mathrm{~g}, 3.64 \mathrm{mmol})$ in DMF $(15 \mathrm{~mL})$ were added imidazole $(800 \mathrm{mg}, 12.3$ mmol) and TBSCl (tert-butylchlorodimethylsilane) (950 $\mathrm{mg}, 7.4 \mathrm{mmol})$. After $18 \mathrm{~h}$ at $25^{\circ} \mathrm{C}$, the reaction mixture was added to $20 \% \mathrm{CH}_{2} \mathrm{Cl}_{2}$ : hexane $(100 \mathrm{~mL})$ and successively washed with $10 \%$ aq. $\mathrm{NaHSO}_{3}(25 \mathrm{~mL})$ and water $(2 \times 25 \mathrm{~mL})$. The organic layer was dried over $\mathrm{Na}_{2} \mathrm{SO}_{4}$ filtered, concentrated in vacuo and distilled to yield $\mathbf{1 2}$ (1.74 $\mathrm{g}, 87 \%)$ as a colorless oil. IR $\left(\mathrm{CHCl}_{3}\right): \vee 3349,3380$, 3088, 2971, 2934, 1699, 1740, 1472, 1452, 1391, 1368, $1311,1256,1152,992,933,771,666,560 \mathrm{~cm}^{-1} ;{ }^{1} \mathrm{H}$ NMR $\left(300 \mathrm{MHz}, \mathrm{CDCl}_{3}\right): \delta 8.10-7.98(\mathrm{~m}, 2 \mathrm{H}), 7.55(\mathrm{t}, J=7.4$ $\mathrm{Hz}, 1 \mathrm{H}), 7.43(\mathrm{t}, J=7.7 \mathrm{~Hz}, 2 \mathrm{H}), 7.28-7.38(\mathrm{~m}, 3 \mathrm{H}), 7.20$ $(\mathrm{d}, J=7.0 \mathrm{~Hz}, 2 \mathrm{H}), 4.6$ (ddt, $J=10.2,6.4,3.1 \mathrm{~Hz}, 1 \mathrm{H})$, $4.33(\mathrm{t}, J=6.5 \mathrm{~Hz}, 2 \mathrm{H}), 4.12-4.27(\mathrm{~m}, 2 \mathrm{H}), 4.02(\mathrm{q}, J=$ $5.4,5.6 \mathrm{~Hz}, 1 \mathrm{H}), 3.88(\mathrm{qd}, J=6.8,1.6 \mathrm{~Hz}, 1 \mathrm{H}), 3.30(\mathrm{dd}$, $J=13.2,3.0 \mathrm{~Hz}, 1 \mathrm{H}), 2.78(\mathrm{dd}, J=13.2,9.6 \mathrm{~Hz}, 1 \mathrm{H}), 1.70$ (m, 2H), 1.62-1.42 (m, 4H), $1.22(\mathrm{~d}, J=6.8 \mathrm{~Hz}, 3 \mathrm{H}), 0.90$ (s, 9H), $0.20(\mathrm{~s}, 6 \mathrm{H}) ;{ }^{13} \mathrm{C}$ NMR $\left(75 \mathrm{MHz}, \mathrm{CDCl}_{3}\right): \delta 175.2$, 166.3, 153.0, 135.0, 133.1, 129.3, 129.1, 128.1, 127.6, 72.6, 65.7, 64.7, 56.3, 43.2, 35.0, 29.2, 26.3, 22.0, 19.0, $12.3,-4.5$; HRMS (ESI): $\mathrm{m} / \mathrm{z}$ calculated for $\mathrm{C}_{31} \mathrm{H}_{47} \mathrm{O}_{6} \mathrm{~N}_{2} \mathrm{Si}$ : $[\mathrm{M}+\mathrm{Na}]^{+}$571.3197, found 571.3174; $[\alpha]_{\mathrm{D}}^{25}=+10.53\left(\right.$ c $\left.1.33, \mathrm{CDCl}_{3}\right)$.

(5S,6S)-5-(tert-Butyldimethylsilyloxy)-7-hydroxy-6methylheptyl benzoate (13): To a stirred solution of $\mathbf{1 2}$ $(1.70 \mathrm{~g}, 3.07 \mathrm{mmol})$ in THF $(70 \mathrm{~mL})$ at $0{ }^{\circ} \mathrm{C}$ was added a solution of $\mathrm{NaBH}_{4}(579 \mathrm{mg}, 15.3 \mathrm{mmol})$ in $\mathrm{pH} 7$ buffer $(18.5 \mathrm{~mL})$. The resulting solution was stirred for $10 \mathrm{~min}$ at $0{ }^{\circ} \mathrm{C}$ before being allowed to gradually warm to room temperature and continued stirring for overnight. The reaction was quenched by the addition of sat. aq. $\mathrm{NH}_{4} \mathrm{Cl}$ $(20 \mathrm{~mL})$ and stirred at room temperature for $1 \mathrm{~h}$. The separated aqueous phase was extracted with EtOAc $(2 \times 25$ mL). The combined organic phase was washed with brine, dried over anhydrous $\mathrm{Na}_{2} \mathrm{SO}_{4}$, filtered and concentrated under reduced pressure. Flash chromatography over silica gel (15\% ethyl acetate in pet. ether) gave $\mathbf{1 3}(1.01 \mathrm{~g}, 87 \%)$ as a colorless oil. IR $\left(\mathrm{CHCl}_{3}\right): \vee 3436,2954,2930,2857$, $1721,1459,1275,1113,1033,836,773,712 \mathrm{~cm}^{-1} ;{ }^{1} \mathrm{H}$ NMR $\left(300 \mathrm{MHz}, \mathrm{CDCl}_{3}\right): \delta 8.02-8.10(\mathrm{~m}, 2 \mathrm{H}), 7.55(\mathrm{t}, J$ $=7.3 \mathrm{~Hz}, 1 \mathrm{H}), 7.43(\mathrm{t}, J=7.9 \mathrm{~Hz}, 2 \mathrm{H}), 4.70(\mathrm{~s}, 1 \mathrm{H}), 4.33$ $(\mathrm{t}, J=6.7 \mathrm{~Hz}, 2 \mathrm{H}), 3.79-3.75(\mathrm{~m}, 1 \mathrm{H}), 3.70(\mathrm{t}, J=9.0 \mathrm{~Hz}$, $2 \mathrm{H}), 3.56-3.48(\mathrm{~m}, 1 \mathrm{H}), 1.97(\mathrm{~m}, 1 \mathrm{H}), 1.56-1.32(\mathrm{~m}$, $6 \mathrm{H}), 0.90(\mathrm{~s}, 9 \mathrm{H}), 0.82(\mathrm{~d}, J=7.8 \mathrm{~Hz}, 3 \mathrm{H}), 0.20(\mathrm{~s}, 6 \mathrm{H})$; ${ }^{13} \mathrm{C} \mathrm{NMR}\left(75 \mathrm{MHz}, \mathrm{CDCl}_{3}\right.$ ): $\delta 166.7,132.8,130.1,129.3$, 128.4, 77.4, 65.5, 64.8, 39.4, 32.0, 28.7, 25.9, 22.7, 17.9, 12.1, -4.6; HRMS (ESI): $\mathrm{m} / z$ calculated for $\mathrm{C}_{21} \mathrm{H}_{37} \mathrm{O}_{4} \mathrm{Si}$ : $[\mathrm{M}+\mathrm{H}]^{+}$381.24556, found 381.24414.

(2R,3S)-7-(Benzoyloxy)-3-(tert-butyldimethylsilyloxy)2-methylheptanoic acid (7): To a stirred solution of $\mathbf{1 3}$ $(1.0 \mathrm{~g}, 2.62 \mathrm{mmol})$ in $\mathrm{CH}_{2} \mathrm{Cl}_{2}(5 \mathrm{~mL})$ at $0{ }^{\circ} \mathrm{C}$ were added 
BAIB ( $3 \mathrm{~g}, 9.31 \mathrm{mmol})$, catalytic amount of TEMPO in p$\mathrm{H} 7$ buffer $(3 \mathrm{~mL})$. The resulting solution was stirred for $10 \mathrm{~min}$ at $0{ }^{\circ} \mathrm{C}$ before being allowed to gradually warm to room temperature with stirring for $1 \mathrm{~h}$. The reaction was quenched by the addition of sat. aq. $\mathrm{NH}_{4} \mathrm{Cl}(20 \mathrm{~mL})$ and stirred at room temperature for $1 \mathrm{~h}$. The separated aqueous phase was extracted with EtOAc $(2 \times 25 \mathrm{~mL})$. The combined organic phase was washed with brine, dried over anhydrous $\mathrm{Na}_{2} \mathrm{SO}_{4}$ filtered and concentrated under reduced pressure. Flash chromatography over silica gel $(30 \%$ ethyl acetate in pet. ether) gave $7(0.92 \mathrm{~g}, 89 \%)$ as a colorless oil. IR $\left(\mathrm{CHCl}_{3}\right): v 2931,2857,1715,1459,1386$, 1274, 1220, 1110, 1069, 1026, 936, 836, 773, 711, 675 $\mathrm{cm}^{-1} ;{ }^{1} \mathrm{H}$ NMR (300 MHz, $\left.\mathrm{CDCl}_{3}\right): \delta 8.10-7.98(\mathrm{~m}, 2 \mathrm{H})$, $7.50(\mathrm{t}, J=7.4 \mathrm{~Hz}, 1 \mathrm{H}), 7.43(\mathrm{t}, J=7.5 \mathrm{~Hz}, 2 \mathrm{H}), 4.33(\mathrm{t}$, $J=6.7 \mathrm{~Hz}, 2 \mathrm{H}), 3.99(\mathrm{q}, J=6.0,5.2 \mathrm{~Hz}, 1 \mathrm{H}), 2.66-2.56$ $(\mathrm{m}, 1 \mathrm{H}), 1.72-1.30(\mathrm{~m}, 6 \mathrm{H}), 1.22(\mathrm{~d}, J=6.8 \mathrm{~Hz}, 3 \mathrm{H}), 0.90$ (s, 9H), $0.20(\mathrm{~s}, 6 \mathrm{H}) ;{ }^{13} \mathrm{C} \mathrm{NMR}\left(75 \mathrm{MHz}, \mathrm{CDCl}_{3}\right): \delta 165.6$, 134.2, 130.0, 129.9, 128.4, 73.5, 65.3, 44.5, 33.5, 29.2, 26.2, 22.2, 18.4, 11.5, -5.4; HRMS (ESI): $\mathrm{m} / \mathrm{z}$ calculated for $\mathrm{C}_{21} \mathrm{H}_{34} \mathrm{O}_{5} \mathrm{Si}:[\mathrm{M}+\mathrm{H}]^{+}$393.4556, found 392.4414.

(2S,3S)-Allyl-2-(tert-butoxycarbonyl(methyl)amino)-3methylpentanoate (15): $\mathrm{Na}_{2} \mathrm{CO}_{3}(2.55 \mathrm{~g}, 24.3 \mathrm{mmol})$ and $\mathrm{Boc}_{2} \mathrm{O}(3.94 \mathrm{~g}, 18.2 \mathrm{mmol})$ were added to a solution of $L$ isoleucine $(3.5 \mathrm{~g}, 12.3 \mathrm{mmol})$, in $\mathrm{H}_{2} \mathrm{O}(20 \mathrm{~mL})$ and THF $(5 \mathrm{~mL})$ at $0{ }^{\circ} \mathrm{C}$. After the reaction mixture has been stirred at room temperature for $12 \mathrm{~h}$, it was neutralized with $\mathrm{HCl}(10 \%)$ until $\mathrm{pH} 2$ has been reached. The mixture was then extracted with EtOAc $(3 \times 50 \mathrm{~mL})$, washed with brine, dried over $\mathrm{Na}_{2} \mathrm{SO}_{4}$. Concentration gave the crude $\mathrm{N}$-Boc-isoleucine $(2.6 \mathrm{~g}, 100 \%)$. $\mathrm{NaH}(60 \%$ in mineral oil, $2.45 \mathrm{~g}, 61.3 \mathrm{mmol}$ ) was added in portions to a solution of $N$-Boc-isoleucine $(2.6 \mathrm{~g}, 9.09 \mathrm{mmol})$ and $\mathrm{MeI}(6.05$ $\mathrm{mL})$ in THF $(50 \mathrm{~mL})$ at $0{ }^{\circ} \mathrm{C}$. After the reaction mixture has been stirred at room temperature for $36 \mathrm{~h}$, it was poured into saturated $\mathrm{NH}_{4} \mathrm{Cl}$ solution $(250 \mathrm{~mL})$, extracted with EtOAc $(3 \times 150 \mathrm{~mL})$ and dried over $\mathrm{Na}_{2} \mathrm{SO}_{4}$. Concentration gave $N$-methyl- $N$-Boc-isoleucine $(2.53 \mathrm{~g}$, $92 \%) . \mathrm{K}_{2} \mathrm{CO}_{3}(3.08 \mathrm{~g}, 22.3 \mathrm{mmol})$ and allyl bromide (1.4 $\mathrm{mL}, 16.9 \mathrm{mmol}$ ) were added to a solution of $N$-methyl $-\mathrm{N}$ Boc-isoleucine $(2.53 \mathrm{~g}, 10.9 \mathrm{mmol})$ in DMSO $(40 \mathrm{~mL})$. After the mixture has been stirred at room temperature for $12 \mathrm{~h}$, it was portioned between EtOAc $(75 \mathrm{~mL})$ and brine $(75 \mathrm{~mL})$. The organic phase was separated and aqueous phase was extracted with EtOAc $(2 \times 100 \mathrm{~mL})$. The combined organic phase was dried over $\mathrm{Na}_{2} \mathrm{SO}_{4}$ and concentrated. Flash chromatography gave $\mathbf{1 5}(2.5 \mathrm{~g}, 80.9 \%)$. IR $\left(\mathrm{CHCl}_{3}\right): \vee 3089,2971,2936,2880,1741,1701,1650$, 1480, 1456, 1393, 1367, 1313, 1255, 1183, 1145, 1047, 990, 931, 871, $773 \mathrm{~cm}^{-1} ;{ }^{1} \mathrm{H}$ NMR (300 MHz, $\left.\mathrm{CDCl}_{3}\right): \delta$ 5.97-5.84 (m, 1H), 5.34-5.20 (m, 1H), 4.62-4.60 (m, $2 \mathrm{H}), 4.55(\mathrm{~d}, J=11.1 \mathrm{~Hz}, 1 \mathrm{H}), 4.26^{*}(\mathrm{~d}, J=11.1 \mathrm{~Hz}, 1 \mathrm{H})$, $2.81(\mathrm{~s}, 3 \mathrm{H}), 2.78^{*}(\mathrm{~s}, 3 \mathrm{H}), 2.04-1.95(\mathrm{~m}, 1 \mathrm{H}), 1.45(\mathrm{~m}$, $10 \mathrm{H}), 1.13-1.01(\mathrm{~m}, 1 \mathrm{H}), 0.92(\mathrm{~d}, J=6.0 \mathrm{~Hz}, 3 \mathrm{H}), 0.87$ $(\mathrm{d}, J=7.2 \mathrm{~Hz}, 3 \mathrm{H}) ;{ }^{13} \mathrm{C} \mathrm{NMR}\left(75 \mathrm{MHz}, \mathrm{CDCl}_{3}\right): \delta 172.3$,
$156.4,132.1,118.4,80.3,65.6,63.8,33.5,30.8,28.7$, 24.5, 15.6, 10.4; HRMS (ESI): $\mathrm{m} / \mathrm{z}$ calculated for $\mathrm{C}_{15} \mathrm{H}_{28} \mathrm{O}_{4} \mathrm{~N}:[\mathrm{M}+\mathrm{H}]^{+}$286.20128, found 286.19969. $[\alpha]_{\mathrm{D}}{ }^{25}$ $=-74.7\left(c 1.1, \mathrm{CHCl}_{3}\right)$. ${ }^{*}$ denotes the rotamer peaks.

(2S,3S)-Allyl-2-((S)-2-(tert-butoxycarbonylamino)- $N, 3$ -dimethylbutanamido)-3-methylpentanoate (8): A solution of (tert-butoxycarbonyl)- $L$-valine $(0.35 \mathrm{~g}, 1.22$ mmol), HATU (0.24 g, $1.8 \mathrm{mmol})$ and HOAt (0.34 g, 1.8 mmol) in $\mathrm{CH}_{2} \mathrm{Cl}_{2}(5 \mathrm{~mL})$ was stirred at $0{ }^{\circ} \mathrm{C}$ under $\mathrm{N}_{2}$ atmosphere for $15 \mathrm{~min}$, treated sequentially with salt [prepared from $15(375 \mathrm{mg}, 1.24 \mathrm{mmol})$ in dry $\mathrm{CH}_{2} \mathrm{Cl}_{2}(1 \mathrm{~mL})$ at $0{ }^{\circ} \mathrm{C}$ on treatment with $\left.\mathrm{CF}_{3} \mathrm{COOH}(0.1 \mathrm{~mL})\right]$ and DIPEA $(0.6 \mathrm{~mL}, 3.6 \mathrm{mmol})$ and stirred for $8 \mathrm{~h}$. The reaction mixture was quenched with aq. satd. $\mathrm{NH}_{4} \mathrm{Cl}$ solution $(10 \mathrm{~mL})$. After $10 \mathrm{~min}$, it was diluted with $\mathrm{CHCl}_{3}(2 \times 10$ $\mathrm{mL})$ and washed with water $(10 \mathrm{~mL}), \mathrm{NaHCO}_{3}$ solution $(10 \mathrm{~mL})$ and brine $(10 \mathrm{~mL})$. The organic layers were dried over $\mathrm{Na}_{2} \mathrm{SO}_{4}$, evaporated and the residue was purified by column chromatography (60-120 mesh silica gel, 35\% ethyl acetate in pet. ether) to afford $\mathbf{8}(509 \mathrm{mg}, 84 \%)$ as a colorless syrup. IR $\left(\mathrm{CHCl}_{3}\right)$ : v 3333, 2966, 2945, 2862, 1742, 1706, 1658, 1462, 1367, 1294, 1178, 1001, 938, $876,774 \mathrm{~cm}^{-1} ;{ }^{1} \mathrm{H}$ NMR $\left(300 \mathrm{MHz}, \mathrm{CDCl}_{3}\right): \delta 5.40-5.41$ $(\mathrm{m}, 1 \mathrm{H}), 5.05(\mathrm{~d}, J=2.2 \mathrm{~Hz}, 2 \mathrm{H}), 4.40(\mathrm{q}, J=2.6 \mathrm{~Hz}, 1 \mathrm{H})$, $3.70(\mathrm{~s}, 3 \mathrm{H}), 3.70-3.50(\mathrm{~m}, 1 \mathrm{H}), 3.50-3.10(\mathrm{~m}, 2 \mathrm{H}), 3.09$ (s, 2H), 2.10-1.89 (m, 2H), $1.50(\mathrm{~s}, 9 \mathrm{H}), 1.32-1.10(\mathrm{~m}$, 5H), $1.00-0.80(\mathrm{~m}, 8 \mathrm{H}) ;{ }^{13} \mathrm{C}$ NMR $\left(75 \mathrm{MHz}, \mathrm{CDCl}_{3}\right): \delta$ 173.5, 171.4, 156.4, 118.6, 79.8, 60.4, 55.3, 52.2, 33.4, 31.4, 29.8, 24.5, 19.8, 16.4, 11.2; HRMS (ESI): $\mathrm{m} / \mathrm{z}$ calculated for $\mathrm{C}_{20} \mathrm{H}_{36} \mathrm{~N}_{2} \mathrm{O}_{5} \mathrm{Na}$ : $[\mathrm{M}+\mathrm{Na}]^{+} 407.2562$, found 407.2414.

$(5 S, 6 R, 9 R, 12 S)$-Allyl-5-(4-(benzoyloxy)butyl)-12-secbutyl-9-isopropyl-2,2,3,3,6,11-hexamethyl-7,10-dioxo4-oxa-8,11-diaza-3-silatridecan-13-oate (16): A solution of acid 7 (510 mg, $1.29 \mathrm{mmol})$, HATU (240 mg, 1.8 mmol) and HOAt (340 mg, $1.8 \mathrm{mmol})$ in $\mathrm{CH}_{2} \mathrm{Cl}_{2}(5 \mathrm{~mL})$ was stirred at $0{ }^{\circ} \mathrm{C}$ under $\mathrm{N}_{2}$ atmosphere for $15 \mathrm{~min}$, treated sequentially with TFA salt [prepared from $8(450 \mathrm{mg}$, $1.2 \mathrm{mmol})$ in dry $\mathrm{CH}_{2} \mathrm{Cl}_{2}(1 \mathrm{~mL})$ at $0{ }^{\circ} \mathrm{C}$ on treatment with $\left.\mathrm{CF}_{3} \mathrm{COOH}(0.1 \mathrm{~mL})\right]$ and DIPEA $(0.6 \mathrm{~mL}, 3.6 \mathrm{mmol})$ and stirred for $8 \mathrm{~h}$. The reaction mixture was quenched with aq. satd. $\mathrm{NH}_{4} \mathrm{Cl}$ solution $(10 \mathrm{~mL})$. After $10 \mathrm{~min}$, it was diluted with $\mathrm{CHCl}_{3}(2 \times 10 \mathrm{~mL})$ and washed with water $(10$ $\mathrm{mL}), \mathrm{NaHCO}_{3}$ solution $(10 \mathrm{~mL})$ and brine $(10 \mathrm{~mL})$. The organic layers were dried over $\mathrm{Na}_{2} \mathrm{SO}_{4}$, evaporated and the residue purified by column chromatography (60-120 mesh silica gel, $45 \%$ ethyl acetate in pet. ether) to afford $16(725 \mathrm{mg}, 85 \%)$ as a colorless syrup. IR $\left(\mathrm{CHCl}_{3}\right): \mathrm{v}$ 3347, 3233, 2966, 2926, 2915, 2854, 2840, 1739, 1701, $1667,1651,1460,1448,1380,1220,1172,1110,1069$, 1026, 1003, 936, 836, 773, 771, 711, 676, $667 \mathrm{~cm}^{-1} ;{ }^{1} \mathrm{H}$ NMR (300 MHz, $\left.\mathrm{CDCl}_{3}\right): \delta 8.00(\mathrm{~d}, J=7.5 \mathrm{~Hz}, 2 \mathrm{H}), 7.55$ $(\mathrm{d}, J=8.3 \mathrm{~Hz}, 1 \mathrm{H}), 7.43(\mathrm{t}, J=7.5 \mathrm{~Hz}, 2 \mathrm{H}), 5.96-5.79$ $(\mathrm{m}, 1 \mathrm{H}), 5.39-5.18(\mathrm{~m}, 2 \mathrm{H}), 5.11-5.01(\mathrm{~m}, 1 \mathrm{H})$, 
4.65-4.54 (m, 2H), 4.34-7.27 (m, 3H), 3.82-3.68 (m, $3 \mathrm{H}), 3.10-3.05(\mathrm{~m}, 2 \mathrm{H}), 2.06-1.89(\mathrm{~m}, 2 \mathrm{H}), 1.83-1.50$ $(\mathrm{m}, 3 \mathrm{H}), 1.45-1.37(\mathrm{~m}, 3 \mathrm{H}), 1.36-1.19(\mathrm{~m}, 4 \mathrm{H})$, $1.20-1.05(\mathrm{~m}, 1 \mathrm{H}), 1.01-0.78(\mathrm{~m}, 22 \mathrm{H}), 0.10-0.03(\mathrm{~m}$, $6 \mathrm{H}) ;{ }^{13} \mathrm{C}$ NMR $\left(75 \mathrm{MHz}, \mathrm{CDCl}_{3}\right): \delta 174.1,171.2,168.4$, $133.5,132.5,130.5,129.2,74.5,66.0,65.3,60.1,58.2$, 54.1, 49.2, 34.2, 32.1, 30.1, 29.4, 26.2, 25.1, 19.2, 16.4, $14.4,11.2,-5.4$. HRMS (ESI): $\mathrm{m} / \mathrm{z}$ calculated for $\mathrm{C}_{36} \mathrm{H}_{60} \mathrm{~N}_{2} \mathrm{O}_{7} \mathrm{SiNa}:[\mathrm{M}+\mathrm{Na}]^{+}$683.4556, found 683.4214.

(5S,6R,9S,12S)-Allyl-12-((S)-sec-butyl)-5-(4-hydroxybutyl)-9-isopropyl-2,2,3,3,6,11-hexamethyl-7,10-dioxo-4-oxa-8,11-diaza-3-silatridecan-13-oate (6): To a stirred solution of $\mathbf{1 6}(700 \mathrm{mg}, 1.06 \mathrm{mmol})$ in $\mathrm{MeOH}(5$ $\mathrm{mL}$ ) was added $\mathrm{K}_{2} \mathrm{CO}_{3}(45 \mathrm{mg}, 0.33 \mathrm{mmol})$. The reaction was stirred at room temperature until complete by TLC ( 2 h). The mixture was then diluted with $\mathrm{CH}_{2} \mathrm{Cl}_{2}(25 \mathrm{~mL})$ and washed with $\mathrm{H}_{2} \mathrm{O}(5 \mathrm{~mL})$. The separated aqueous phase was extracted with $\mathrm{CH}_{2} \mathrm{Cl}_{2}(3 \times 10 \mathrm{~mL})$ and the combined organic phase dried over $\mathrm{Na}_{2} \mathrm{SO}_{4}$, filtered, evaporated and the residue was purified by column chromatography (60-120 mesh silica gel, 52\% ethyl acetate in pet. ether) to afford 6 (440 mg, 75\%) as a colorless syrup. IR $\left(\mathrm{CHCl}_{3}\right): \vee 3347,3300,3233,2956,2926,2925,2854$, 2840, 1701, 1667, 1651, 1460, 1448, 1380, 1220, 1172, 1110, 1069, 1026, 1003, 936, 836, 773, 771, 711, 676, $667 \mathrm{~cm}^{-1} ;{ }^{1} \mathrm{H}$ NMR $\left(300 \mathrm{MHz}, \mathrm{CDCl}_{3}\right): \delta 5.96-5.79(\mathrm{~m}$, $1 \mathrm{H}), 5.39-5.18(\mathrm{~m}, 2 \mathrm{H}), 5.11-5.01(\mathrm{~m}, 1 \mathrm{H}), 4.65-4.54$ (m, 2H), 4.50 (brs, $1 \mathrm{H}), 4.34-4.27(\mathrm{~m}, 3 \mathrm{H}), 4.25-4.15(\mathrm{~m}$, $2 \mathrm{H}), 3.68(\mathrm{~m}, 3 \mathrm{H}), 3.10-3.05(\mathrm{~m}, 2 \mathrm{H}), 2.06-1.89(\mathrm{~m}, 2 \mathrm{H})$, $1.83-1.50(\mathrm{~m}, 3 \mathrm{H}), 1.45-1.37(\mathrm{~m}, 3 \mathrm{H}), 1.36-1.19(\mathrm{~m}$, $4 \mathrm{H}), 1.20-1.05(\mathrm{~m}, 1 \mathrm{H}), 1.01-0.78(\mathrm{~m}, 19 \mathrm{H}), 0.10-0.03$ $(\mathrm{m}, 6 \mathrm{H}) ;{ }^{13} \mathrm{C}$ NMR $\left(75 \mathrm{MHz}, \mathrm{CDCl}_{3}\right): \delta 174.1,171.2$, $132.5,74.5,66.0,65.3,62.8,60.1,58.2,54.1,49.2,34.2$, 32.1, 30.1, 29.4, 26.2, 25.1, 19.2, 16.4, 14.4, 11.2, -5.4; HRMS (ESI): $\mathrm{m} / \mathrm{z}$ calculated for $\mathrm{C}_{29} \mathrm{H}_{56} \mathrm{~N}_{2} \mathrm{O}_{6} \mathrm{SiNa}$ : $[\mathrm{M}+\mathrm{Na}]^{+} 579.3914$, found 579.3812 .

$(5 S, 6 R, 9 S, 12 S)$-Allyl 12-((S)-sec-butyl)-9-isopropyl2,2,3,3,6,11-hexamethyl-7,10-dioxo-5-(pent-4-ynyl)-4oxa-8, 11-diaza-3-silatridecan-13-oate (5): To a stirred solution of 6 (420 mg, $0.755 \mathrm{mmol})$ and powdered $4 \AA$ molecular sieves $(650 \mathrm{mg})$ in $\mathrm{CH}_{2} \mathrm{Cl}_{2}(15 \mathrm{~mL})$ were subsequently added 4-methylmorpholine- $N$-oxide $(227 \mathrm{mg}$, $1.94 \mathrm{mmol})$ and tetrapropylammonium perruthenate (22.9 $\mathrm{mg}, 0.065 \mathrm{mmol}$ ) and the mixture was stirred at room temperature for $2 \mathrm{~h}$. The reaction mixture was filtered through a pad of silica gel and the filtrate was concentrated under reduced pressure to give the aldehyde as a colorless oil. The residue was placed under high vaccum for $2 \mathrm{~h}$ before being used in the subsequent reaction without purifiation. The aldehyde, $\mathrm{K}_{2} \mathrm{CO}_{3}$ and the Ohira-Bestmen reagent $(262 \mathrm{mg}, 1.32 \mathrm{mmol})$ were stirred for $16 \mathrm{~h}$. The mixture was then diluted with $\mathrm{CH}_{2} \mathrm{Cl}_{2}(25 \mathrm{~mL})$ and washed with $\mathrm{H}_{2} \mathrm{O}(5 \mathrm{~mL})$. The separated aqueous phase was extracted with $\mathrm{CH}_{2} \mathrm{Cl}_{2}(3 \times 10 \mathrm{~mL})$ and the combined orga- nic phase dried over $\mathrm{Na}_{2} \mathrm{SO}_{4}$, filtered and evaporated and the residue was purified by column chromatography (60-120 mesh silica gel, 5\% ethyl acetate in pet. ether) to afford 5 (352 $\mathrm{mg}, 92 \%)$ as a yellow syrup. ${ }^{1} \mathrm{H}$ NMR(300 $\left.\mathrm{MHz}, \mathrm{CDCl}_{3}\right): \delta 5.86-5.74(\mathrm{~m}, 1 \mathrm{H}), 5.29-5.08(\mathrm{~m}, 2 \mathrm{H})$, 5.04-5.01 $(\mathrm{m}, 1 \mathrm{H}), 4.45-4.34(\mathrm{~m}, 2 \mathrm{H}), 4.14-4.07(\mathrm{~m}$, $3 \mathrm{H}), 4.02-3.95(\mathrm{~m}, 2 \mathrm{H}), 3.58(\mathrm{~m}, 3 \mathrm{H}), 3.10-3.05(\mathrm{~m}, 2 \mathrm{H})$, $2.20(\mathrm{~s}, 1 \mathrm{H}), 2.06-1.89(\mathrm{~m}, 2 \mathrm{H}), 1.83-1.50(\mathrm{~m}, 3 \mathrm{H})$, 1.45-1.37 (m, 3H), 1.36-1.19 (m, 4H), 1.20-1.05 (m, 1H), $1.01-0.78(\mathrm{~m}, 19 \mathrm{H}), 0.10-0.03(\mathrm{~m}, 6 \mathrm{H}) ;{ }^{13} \mathrm{C} \mathrm{NMR}$ $\left(75 \mathrm{MHz}, \mathrm{CDCl}_{3}\right): \delta 172.1,170.2,129.5,83.9,71.5,68.2$, 61.3, 60.8, 60.1, 53.2, 52.1, 48.2, 33.2, 32.1, 30.1, 29.4, 26.2, 25.1, 18.7, 16.4, 14.4, 11.2, -5.4; HRMS (ESI): $\mathrm{m} / \mathrm{z}$ calculated for $\mathrm{C}_{30} \mathrm{H}_{54} \mathrm{~N}_{2} \mathrm{O}_{5} \mathrm{SiNa}:[\mathrm{M}+\mathrm{H}]^{+}$551.3140, found 579.3120 .

\section{Conclusions}

In conclusion, a practical and stereoselective synthesis of C21-C41 fragment of hantupeptin A having five stereo centers, two amide linkages and one ester linkage was demonstrated with differential protective groups to allow further extensions. The key features of the strategy are the successful utilization of Evan's Aldol reaction, TEMPO mediated oxidation and Ohira-Bestmann homologation. Furthur investigation towards the total synthesis of hantupeptin A are in progress.

\section{Acknowledgements}

A. Srinivas is thankful to CSIR, New Delhi for Research Associate fellowship, Director, Indian Institute of Chemical Technology (IICT), Hyderabad for the providing research facilities and Dr. G. V. M. Sharma, Chief Scientist, IICT, Hyderabad for valuable guidance.

\section{References}

1. A. Yurek-George, A. R. Cecil, A. H. Mo, S. Wen, H. Rogers, F. Habens, S. Maeda, M. Yoshida, G. Packham, A. Ganesan, J. Med. Chem. 2007, 50, 5720-5726. http://dx.doi.org/10.1021/jm0703800

2. J. Adrio, C. Cuevas, I. Manzanares, M. M. Joullié, J. Org. Chem. 2007, 72, 5129-5138.

http://dx.doi.org/10.1021/jo070412r

3. B. Liang, D. J. Richard, P. S. Portonovo, M. M. Joullie, J. Am. Chem. Soc. 2001, 123, 4469-4474.

http://dx.doi.org/10.1021/ja010222c

4. P. S. Pan, K. L. McGuire, S. R. McAlpine, Bioorg. Med. Chem. Lett. 2007, 17, 5072-5077.

http://dx.doi.org/10.1016/j.bmcl.2007.07.025

5. K. Otrubova, G. Lushington, D. V. Velde, K. L. McGuire, S. R. McAlpine, J. Med. Chem. 2008, 51, 530-544. 
http://dx.doi.org/10.1021/jm070731a

6. R. Lemmens-Gruber, M. R. Kamyar, R. Dornetshuber, Curr. Med. Chem. 2009, 16, 1122-1137.

http://dx.doi.org/10.2174/092986709787581761

7. F. Sarabia, S. Chammaa, A. S. Ruiz, L. M. Ortiz, F. J. Herrera, Curr. Med. Chem. 2004, 11, 1309-1332. http://dx.doi.org/10.2174/0929867043365224

8. M. D. Vera, M. M. Joullie, Med. Res. Rev. 2002, 22, 102145. http://dx.doi.org/10.1002/med.10003

9. A. Tripathi, J. Puddick, M. R. Prinsep, P. P. F. Lee, L. T. Tan, J. Nat. Prod. 2009, 72, 29-32. http://dx.doi.org/10.1021/np800448t

10. A. Tripathi, J. Puddick, M. R. Prinsep, P. P. F. Lee, L. T. Tan, Phytochemistry 2010, 71, 307-311. http://dx.doi.org/10.1016/j.phytochem.2009.10.006

11. D. K. Gupta, G. C. Ding, Y. C. Teo, L. T. Tan, Nat. Prod. Commun. 2016, 11, 69-72.

12. (a) A. Srinivas, A. Nagaraj, C. S. Reddy, Eur. J. Med. Chem. 2010, 45, 2353-2358. http://dx.doi.org/10.1016/j.ejmech.2010.02.014

(b) C. S. Reddy, A. Srinivas, M. Sunitha, A. Nagaraj, Heterocycl. Chem. 2010, 47, 1303-130. http://dx.doi.org/10.1002/jhet.474

(c) C. S. Reddy, A. Nagaraj, A. Srinivas, G. P. Reddy, Indian J. Chem. 2010, 49B, 617-622.

(d) A. Srinivas, C. S. Reddy, A. Nagaraj, Chem. Pharm. Bull. 2009, 57, 685-693. http://dx.doi.org/10.1248/cpb.57.685

(e) C. S. Reddy, A. Srinivas, A. Nagaraj, J. Heterocyclic. Chem. 2009, 46, 497-502. (f) C. S. Reddy, A. Nagaraj, A. Srinivas, G. P. Reddy, Indian J. Chem. 2009, 48B, 248-254. (g) C. S. Reddy, A. Srinivas, A. Nagaraj, J. Heterocyclic. Chem. 2008, 45, 999-1003. (h) C. S. Reddy, A. Srinivas, A. Nagaraj, J. Heterocyclic. Chem. 2008, 45, 1121-1125. (i) C. S. Reddy, G. P. Reddy, A. Nagaraj, A. Srinivas, Org. Commun. 2008, 1, 84-94. (j) C.S Reddy, A. Srinivas, A. Nagaraj, Indian J. Chem. 2008, 47B, 787-791.

13. K. Kubota, E. Yamamoto, H. Ito, J. Am. Chem. Soc. 2015, 137, 420-424. http://dx.doi.org/10.1021/ja511247z

14. A. D. Fotiadou, A. L. Zografos, Org. Lett. 2011, 13, 45924595. http://dx.doi.org/10.1021/ol2017802

15. J. R. Gage, D. A. Evans, Org. Synth. 1990, 68, 83-91. http://dx.doi.org/10.15227/orgsyn.068.0083

\section{Povzetek}

Opisana je stereoselektivna sinteza južnega fragmenta (C21-C41) hantupeptina A. Zahtevana stereokemija $\beta$-hidroksi$\alpha$-metil kislinske enote je bile dosežen z aldolno reakcijo z uporabo Evansovega kiralnega pomagala, ki ji je sledila uvedba terminalnega alkinskega ostanka s pomočjo Ohira-Bestmannovega reagenta. 